\title{
The association between physical activity and bladder cancer: systematic review and meta-analysis
}

\author{
M Keimling ${ }^{*}{ }^{*}$, G Behrens ${ }^{1}$, D Schmid ${ }^{1}$, C Jochem $^{1}$ and M F Leitzmann ${ }^{1}$ \\ ${ }^{1}$ Department of Epidemiology and Preventive Medicine, University of Regensburg, Franz-Josef-Strauss-Allee 11, 93053 \\ Regensburg, Germany
}

Background: Physical activity may protect against bladder cancer through several biologic pathways, such as enhanced immune function and decreased chronic inflammation. Physical activity may also indirectly prevent bladder cancer by reducing obesity. A sizeable number of epidemiologic studies have examined the association between physical activity and bladder cancer, but the available evidence has not yet been formally summarised using meta-analysis.

Methods: We performed a systematic literature review and meta-analysis of English-language studies published from January 1975 through November 2013. We followed the PRISMA guidelines and used a random effects model to estimate the summary risk estimates for the association between physical activity and bladder cancer.

Results: A total of 15 studies with 5402369 subjects and 27784 bladder cancer cases were included. High vs low levels of physical activity were related to decreased bladder cancer risk (summary relative risk $(R R)=0.85$, 95\% confidence interval $(C I)=0.74-0.98$; $P^{2}=83 \%$; $P$-value for heterogeneity across all studies $\left.<0.001\right)$. Results were similar for cohort studies $(R R=0.89,95 \% \mathrm{Cl}=0.80-1.00$; $\left.P^{2}=64 \%\right)$ and case-control studies $\left(R R=0.71,95 \% \mathrm{Cl}=0.43-1.16 ; P^{2}=87 \% ; P\right.$-value for difference $\left.=0.108\right)$ and they were comparable for women $\left(\mathrm{RR}=0.83,95 \% \mathrm{Cl}=0.73-0.94 ; P^{2}=0 \%\right.$ ) and men (RR=0.92,95\% Cl=0.82-1.05; $P^{2}=67 ; P$-value for difference $=0.657)$. Findings were also comparable for recreational $\left(R R=0.81,95 \% \mathrm{Cl}=0.66-0.99 ; P^{2}=77 \%\right)$ and occupational physical activity (RR $=0.90,95 \% \mathrm{Cl}=0.76-1.0 ; P^{2}=76 \% ; P$-value for difference $\left.=0.374\right)$, and they were largely consistent for moderate $\left(\mathrm{RR}=0.85,95 \% \mathrm{Cl}=0.75-0.98 ; P^{2}=76 \%\right)$ and vigorous activity $\left(\mathrm{RR}=0.80,95 \% \mathrm{Cl}=0.64-1.00 ; \mathrm{I}^{2}=87 \%\right.$; $P$-value for difference $=0.535$ ).

Conclusions: Physical activity is associated with decreased risk of bladder cancer. Further studies are required to assess the relations of intensity, frequency, duration, and timing in life of physical activity to bladder cancer risk.

During the past 10 years, the incidence rate of bladder cancer has increased by 26\% (Jemal et al, 2003; Siegel et al, 2013). In 2012, approximately 72570 new cases of bladder cancer were diagnosed in the United States (U.S.) (Siegel et al, 2013), and approximately 150000 new cases were diagnosed in 40 European countries (Ferlay et al, 2013). Risk factors for bladder cancer include age, male gender, smoking, exposure to arsenic in drinking water, and occupational exposure to aromatic amines (Bachir and Kassouf, 2012). In addition, obesity appears to modestly increase the risk of bladder cancer (Qin et al, 2013).
In contrast, physical activity may protect against bladder cancer. Potential biologic pathways linking increased physical activity to decreased risk of bladder cancer include enhanced immune function, reduced chronic inflammation, increased detoxification of carcinogens, enhanced DNA repair, and modified cell proliferation, differentiation, and apoptosis (Rogers et al, 2008). Physical activity may also indirectly prevent bladder cancer by reducing obesity and contributing to body weight maintenance (Koebnick et al, 2008).

To date, 15 studies (Severson et al, 1989; Brownson et al, 1991; Paffenbarger et al, 1992; Dosemeci et al, 1993; Wannamethee et al,

*Correspondence: Dr M Keimling; E-mail: marlen.keimling@klinik.uni-regensburg.de

Received 23 August 2013; revised 20 December 2013; accepted 24 December 2013; published online 4 March 2014

(c) 2014 Cancer Research UK. All rights reserved 0007-0920/14 
2001; Tripathi et al, 2002; Soll-Johanning and Bach, 2004; Schnohr et al, 2005; Holick et al, 2007; Koebnick et al, 2008; Wilson et al, 2008; Yun et al, 2008; Lin et al, 2010; Parent et al, 2011; Sormunen et al, 2013) have investigated the association between physical activity and risk of bladder cancer. Seven studies (Paffenbarger et al, 1992; Tripathi et al, 2002; Schnohr et al, 2005; Koebnick et al, 2008; Wilson et al, 2008; Lin et al, 2010; Parent et al, 2011) found an inverse relation between physical activity and bladder cancer, two (Wilson et al, 2008; Lin et al, 2010) of which were statistically significant. In contrast, two studies (Wannamethee et al, 2001; Sormunen et al, 2013) detected a positive association between physical activity and bladder cancer, one (Wannamethee et al, 2001) of which was statistically significant. Six studies (Severson et al, 1989; Brownson et al, 1991; Dosemeci et al, 1993; SollJohanning and Bach, 2004; Holick et al, 2007; Yun et al, 2008) reported a null relation between physical activity and bladder cancer. Despite the availability of a sizeable number of epidemiologic studies and the existence of several plausible biological mechanisms linking increased physical activity to decreased risk of bladder cancer, the evidence has not yet been formally summarised using meta-analysis. Because of this limitation, we performed a comprehensive systematic review and meta-analysis to quantify the relation of physical activity to bladder cancer risk.

\section{MATERIALS AND METHODS}

Literature Search. Our systematic review and meta-analysis followed the PRISMA (Preferred reporting items for systematic reviews and meta-analyses) guidelines consisting of a checklist including 27 items (Moher et al, 2009). We performed a literature search of scientific articles published from January 1975 to November 2013 in the Cochrane Library, EMBASE, MEDLINE, and Web of Science using the following terms for physical activity: 'physical activity', 'motor activity', 'exercise', 'physical fitness', 'endurance training,, 'sport', 'athlete', 'sedentary', 'sedentary lifestyle', 'physical inactivity', 'motor inactivity', 'recreation', 'occupation', 'walking', and 'sitting'. Using an AND operator, we combined those terms with the following terms for bladder cancer outcomes: 'urinary bladder neoplasms', 'bladder cancer', 'bladder carcinoma, 'bladder adenocarcinoma', and 'bladder tumour'. Our literature search strategy focused on human research articles written in English language. In addition, we screened the reference lists of the articles to identify further studies.

Studies were included in the meta-analysis if they (1) investigated the association between physical activity and bladder cancer incidence, (2) were observational studies with a cohort or casecontrol design, (3) published risk estimates such as hazard ratios (HR), odds ratios (OR), relative risks (RR), or standardised incidence ratios (SIR) and 95\% confidence intervals (95\% CI) or sufficient information to calculate them, and (4) provided ageadjusted or age-matched risk estimates. With the exception of one study of physically active mail carriers (Soll-Johanning and Bach, 2004) and one study of athletes (Sormunen et al, 2013), we did not consider occupational cohort studies that were based on record linkage of job titles to bladder cancer because those studies focused on occupational exposure to air pollution.

Two authors (MK, MFL) independently reviewed all identified studies quantifying the association between physical activity and risk of bladder cancer. A detailed overview of our literature search is shown in Supplementary Figure S1.

Our initial literature search yielded 3900 articles, of which 2410 articles remained after a duplicate check. After screening for irrelevant titles ( $n=2310$ articles) and abstracts ( $n=63$ articles), the remaining 37 manuscripts were reviewed in depth. Subsequently, eight studies were found in the reference lists of the reviewed manuscripts. Of these 45 articles, we excluded 28 articles that investigated the association between job titles and bladder cancer without focusing on physical activity. Further, we removed one article (Pukkala et al, 2000) because the results were updated in a subsequent study (Sormunen et al, 2013). We also removed one study that focused on physical activity and bladder cancer mortality (Batty et al, 2010). Thus, 15 articles were included in the metaanalysis (Severson et al, 1989; Brownson et al, 1991; Paffenbarger et al, 1992; Dosemeci et al, 1993; Wannamethee et al, 2001; Tripathi et al, 2002; Soll-Johanning and Bach, 2004; Schnohr et al, 2005; Holick et al, 2007; Koebnick et al, 2008; Wilson et al, 2008; Yun et al, 2008; Lin et al, 2010; Parent et al, 2011; Sormunen et al, 2013).

Data extraction. From each study, we extracted the first author's last name, publication year, location of the study, sample size, number of cases, gender, domains and intensities of physical activity, type of assessment of physical activity, timing in life of physical activity, highest $v s$ lowest category of physical activity, risk estimates with corresponding 95\% confidence intervals (CI), and adjustment variables.

In four studies (Brownson et al, 1991; Dosemeci et al, 1993; Wilson et al, 2008; Lin et al, 2010), the reported risk estimates were based on using the highest level of physical activity as the reference group. For comparability with the other studies, we converted the reported RR to its reciprocal value. In one study (Paffenbarger et al, 1992), we calculated the 95\% CI using the $P$-value and the RR estimate.

When grouping studies by the potential effect modifying factor 'component or measure of physical activity', we created the categories 'energy expenditure', 'activity duration', 'activity frequency', and 'qualitative assessments'. Assessments of energy expenditure included metabolic equivalents of tasks (METs) per week, kilo joule $(\mathrm{kJ}) /$ minute, or weighted physical activity indexes. Activity duration was defined as hours/week or percentage of time spent physically active. Activity frequency was reported as times per week of physical activity. Qualitative assessments of physical activity were based on categories such as 'sedentary', 'light', 'moderate', or 'high' physical activity. In one study (Yun et al, 2008), physical activity levels were expressed as the combination of activity frequency and duration. For comparability with other studies, we grouped that study into the category 'activity duration'.

Statistical analysis. We interpreted hazard ratios and odds ratios as estimates of the $\mathrm{RR}_{\mathrm{i}}$. We computed the natural logarithms of those risk estimates $\log \left(\mathrm{RR}_{\mathrm{i}}\right)$ with their corresponding s.e. $\mathrm{s}_{\mathrm{i}}=$ $(\log ($ upper $95 \%$ CI bound of $\mathrm{RR})-\log (\mathrm{RR})) / 1.96$ and used a random effects model to determine the weighted average of those $\log \left(\mathrm{RR}_{\mathrm{i}}\right)$ s while allowing for effect measure heterogeneity. We weighted the $\log \left(R R_{i}\right) s$ by $w_{i}=1 /\left(s_{i}^{2}+t^{2}\right)$, where $s_{i}$ denoted the standard error of $\log \left(\mathrm{RR}_{\mathrm{i}}\right)$ and $t^{2}$ denoted the restricted maximum likelihood estimate of the overall variance (Higgins and Thompson, 2002). Heterogeneity was assessed using $Q^{-}$and $I^{2}$-statistics (Higgins and Thompson, 2002). We examined publication bias using a funnel plot (Egger et al, 1997), Egger's regression test (Egger et al, 1997), and Begg's rank correlation test (Begg and Mazumdar, 1994). $P$-values were considered statistically significant at the 0.05 level. All statistical analyses were conducted in $\mathrm{R}$ (R Development Core Team, 2011) using the R-package 'metafor' (Viechtbauer, 2010).

The main analysis included one physical activity risk estimate per study. If a study reported risk estimates for men and women separately, we included both risk estimates in the meta-analysis because they were based on independent samples. If a study reported on different domains and/or intensities of physical activity, we included the risk estimate for recreational and vigorous activity.

In a series of subanalyses that were determined a priori, we examined the relation of physical activity to bladder cancer risk within categories of study design (cohort study, case-control study), physical activity domain (recreational, occupational), 


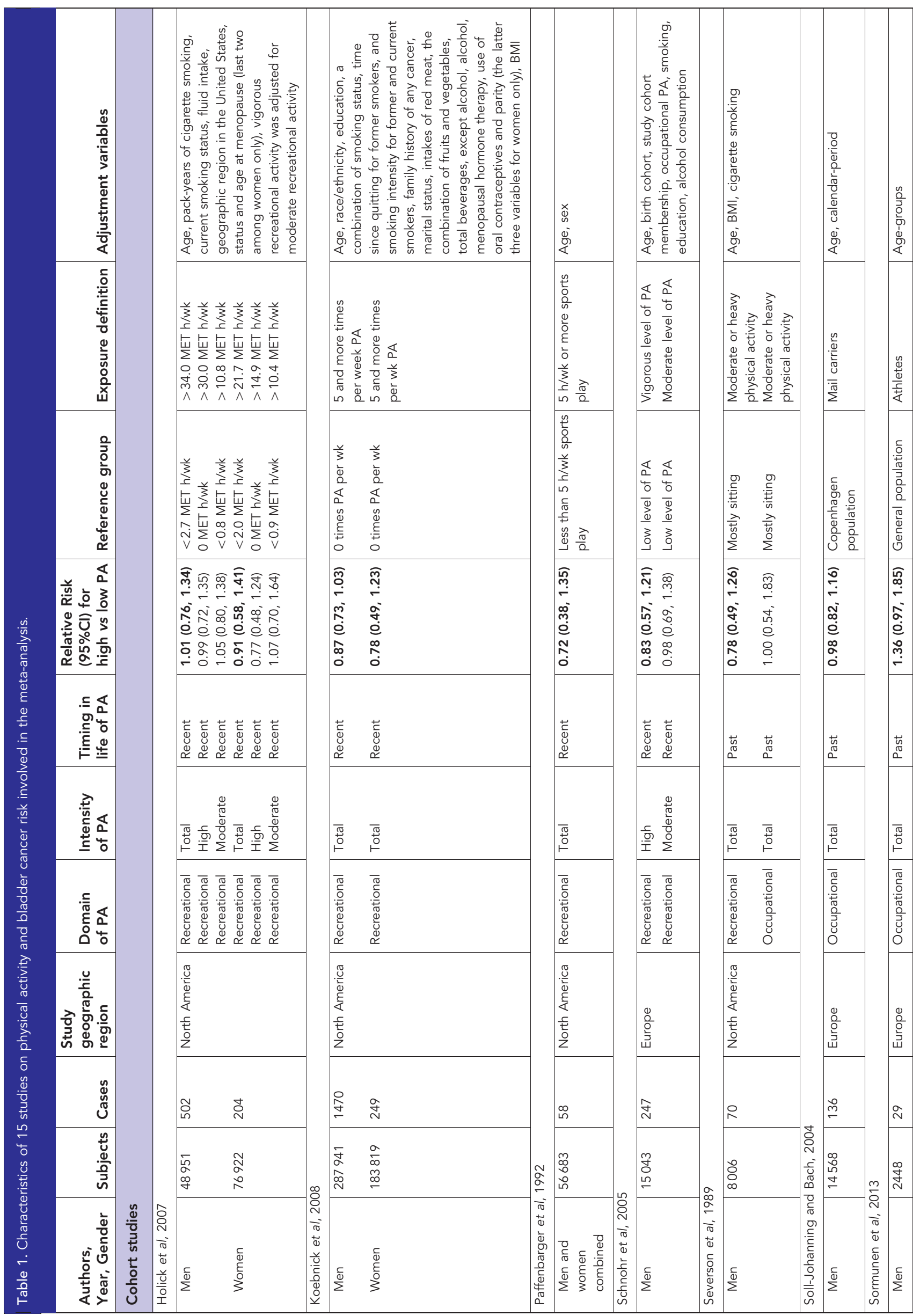




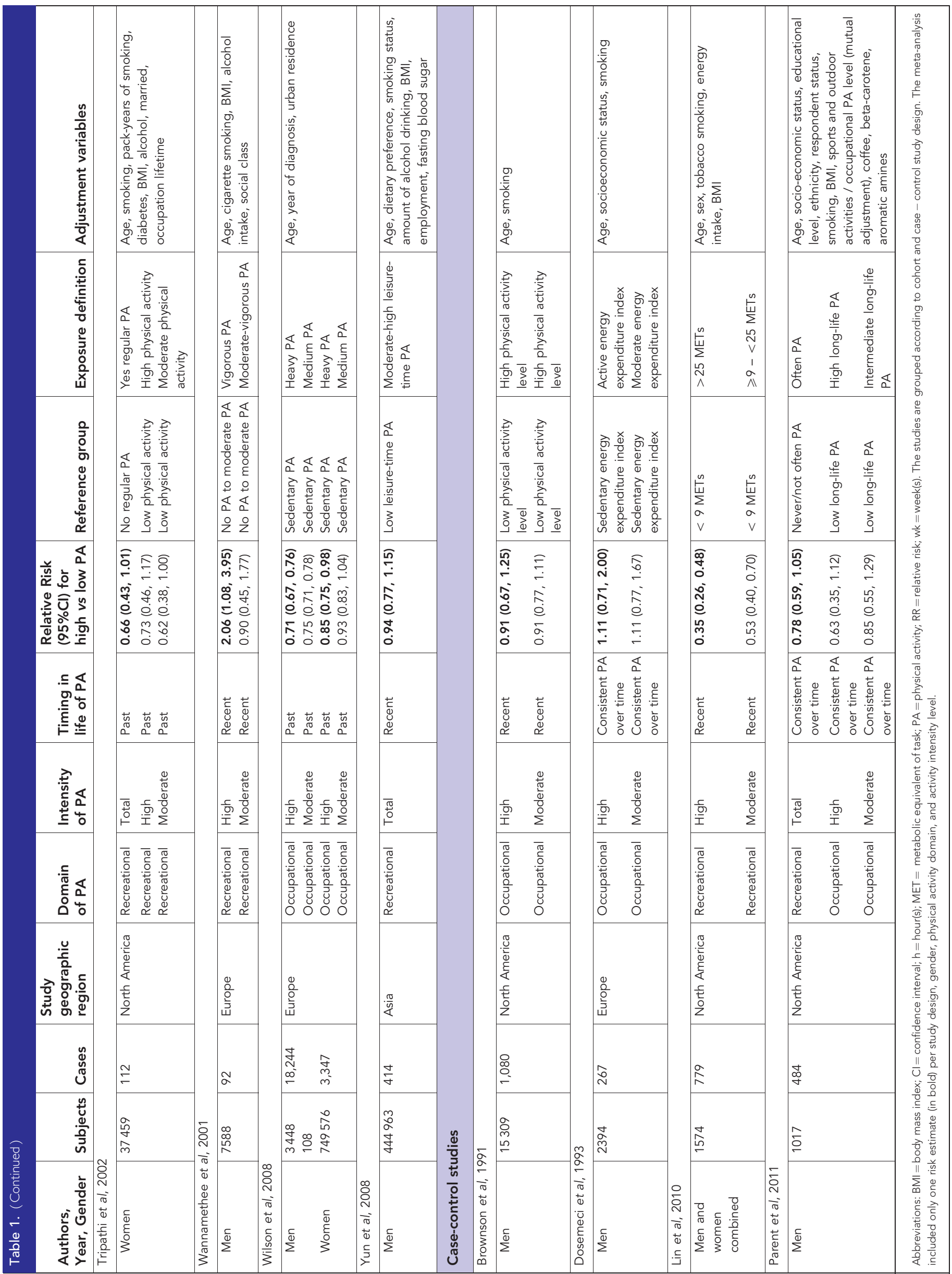


gender (men, women, men, and women combined), physical activity intensity (moderate, vigorous), component or measure of physical activity (energy expenditure, activity duration, activity frequency, qualitative assessments), timing in life of physical activity (recent, consistent over time, past), type of physical activity assessment (interview, self-reported, by proxy), number of adjustment factors (greater or less than the median), adjustment for body mass index (BMI; yes, no), adjustment for smoking (yes, no), and study geographic region (North America, Europe, Asia).

The number of risk estimates included in the stratified analyses differed for each subanalysis. Subanalyses stratified by study design, gender, component or measure of physical activity, timing in life of physical activity, type of physical activity assessment, number of adjustment factors, adjustment for BMI, adjustment for smoking, and study geographic region included 18 risk estimates. The subanalysis stratified by physical activity domain contained 20 risk estimates, and the subanalysis stratified by activity intensity included 22 risk estimates.

We evaluated potential heterogeneity of the physical activity and bladder cancer relation according to those factors by using random effects meta-analysis regression where we compared the model that included the current factor of interest as a single explanatory variable with the null model that included no explanatory variable.

Dose-response analysis. We employed a fractional polynomial approach (Rota et al, 2010) to fit a non-linear dose response metaanalysis using all studies that included a minimum of three physical activity categories (Severson et al, 1989; Brownson et al, 1991; Dosemeci et al, 1993; Wannamethee et al, 2001; Tripathi et al, 2002; Schnohr et al, 2005; Holick et al, 2007; Koebnick et al, 2008; Wilson et al, 2008; Lin et al, 2010; Parent et al, 2011). For each study, we converted the physical activity cut points to percentile cut points based on the reported physical activity group sizes. Percentiles ranged from 0 to 100 , with 0 indicating the lowest physical activity level and 100 indicating the highest physical activity level.

\section{RESULTS}

Study characteristics. The main characteristics of the eleven cohort studies (Severson et al, 1989; Paffenbarger et al, 1992; Wannamethee et al, 2001; Tripathi et al, 2002; Soll-Johanning and Bach, 2004; Schnohr et al, 2005; Holick et al, 2007; Koebnick et al, 2008; Wilson et al, 2008; Yun et al, 2008; Sormunen et al, 2013) and four case - control studies (Brownson et al, 1991; Dosemeci et al, 1993; Lin et al, 2010; Parent et al, 2011) are shown in Table 1. Three studies (Holick et al, 2007; Koebnick et al, 2008; Wilson et al, 2008) presented results stratified by gender, yielding a total of 18 independent risk estimates. A total of 5402369 subjects and 27784 bladder cancer cases were included in the meta-analysis. The majority of risk estimates involved men, were derived from studies located in North America, and were based on recreational activity. Eight studies used self-report questionnaires to assess physical activity, four studies assessed physical activity using an interview, and three studies used information provided by proxy. Five studies used energy expenditure as a measure of physical activity, four studies used data on activity duration, four studies used information on activity frequency, and three studies were based on qualitative measures of physical activity. Eight studies assessed recent physical activity, five studies collected information on past physical activity, and two studies evaluated consistent physical activity over time. The number of adjustment factors in the individual studies ranged between one and fourteen. Eleven studies adjusted for smoking and seven studies adjusted for BMI.

Main analysis. Comparing the highest with the lowest physical activity level, the combined bladder cancer risk estimate was 0.85
(95\% CI $=0.74-0.98)$, with considerable between-study heterogeneity $\left(\mathrm{I}^{2}=83 \%, P\right.$-value for heterogeneity across all studies $<0.001$ ) (Figure 1). After removal of case-control studies (Brownson et al, 1991; Dosemeci et al, 1993; Lin et al, 2010; Parent et al, 2011) and cohort studies that did not control for smoking (Paffenbarger et al, 1992; Soll-Johanning and Bach, 2004; Wilson et al, 2008; Sormunen et al, 2013), the heterogeneity of the data was no longer apparent $\left(I^{2}=0 \%, P\right.$-value for heterogeneity $=0.256)$. No publication bias was demonstrated by Egger's regression test $(P=0.467)$, Begg's rank correlation test $(P=0.654)$, or the funnel plot (Supplementary Figure S2).

Study design. We investigated cohort and case - control studies separately and observed a stronger but statistically non-significant inverse association between physical activity and bladder cancer in case - control studies $(\mathrm{RR}=0.71,95 \% \mathrm{CI}=0.43-1.16)$ than in cohort studies $(\mathrm{RR}=0.89,95 \% \mathrm{CI}=0.80-1.00)$, although that difference was not statistically significant $(P$-value for difference by study design $=0.108$ ) (Figure 1$)$. Between-study heterogeneity was more pronounced in case - control studies $\left(I^{2}=87 \%\right)$ than cohort studies $\left(I^{2}=64 \%\right)$. There was no heterogeneity in cohort studies that adjusted for smoking $\left(I^{2}=0 \%, \quad P\right.$-value for heterogeneity $=0.256$ ), whereas cohort studies that did not adjust for smoking indicated considerable heterogeneity $\left(I^{2}=88 \%\right.$, $P$-value for heterogeneity $<0.001)$.

Physical activity domain. Both recreational and occupational activities were related to decreased risk of bladder cancer, and the relation with recreational activity was statistically significant (Figure 2). The summary risk estimates for recreational and occupational activity were $0.81\left(95 \% \mathrm{CI}=0.66-0.99, I^{2}=77 \%\right)$ and 0.90 (95\% CI $\left.=0.76-1.07, I^{2}=76 \%\right)$, respectively, comparing the highest with the lowest levels of activity ( $P$-value for difference by physical activity domain $=0.374$ ).

Gender. When stratifying by gender, the summary risk estimate appeared to be slightly more pronounced in women $(\mathrm{RR}=0.83$, $95 \% \mathrm{CI}=0.73-0.94)$ than men $(\mathrm{RR}=0.92,95 \% \mathrm{CI}=0.82-1.05)$ but that difference was not statistically significant $(P$-value for difference by gender $=0.657) \quad($ Table 2$)$. We noted sizeable between-study heterogeneity among men $\left(I^{2}=67 \%\right)$ but not among women $\left(I^{2}=0 \%\right)$.

Activity intensity. The magnitude of the inverse relations of moderate and vigorous activity to bladder cancer were comparable, although the summary risk estimate for vigorous physical activity did not reach statistical significance (RR for moderate intensity activity $=0.85,95 \% \mathrm{CI}=0.75-0.98, I^{2}=76 \%$; RR for vigorous intensity activity $=0.80,95 \% \mathrm{CI}=0.64-1.00, I^{2}=87 \%$; $P$-value for difference by activity intensity $=0.535)$ (Table 2$)$.

Other factors. No clear pattern of difference regarding the physical activity and bladder cancer relation emerged in analyses stratified by component or measure of physical activity $(P=0.783)$, timing in life of physical activity $(P=0.962)$, type of physical activity assessment $(P=0.201)$, number of adjustment factors ( $P=0.739)$, adjustment for BMI $(P=0.231)$, adjustment for smoking $(P=0.620)$, or study geographic region $(P=0.217)$ (Table 2). Three studies (Koebnick et al, 2008; Yun et al, 2008; Lin et al, 2010) examined whether the physical activity and bladder cancer relation was modified by smoking status. We pooled the findings from those studies and did not find a significant difference regarding the relation of physical activity to bladder cancer between current, former, or never smokers ( $\mathrm{p}$-difference by smoking status $=0.846$ ).

Dose-response meta-analysis. The dose-response meta-analysis revealed an approximately linear relation between physical activity percentile and bladder cancer risk. The 25th, 50th, and 75th physical activity level percentiles were associated with reductions in 


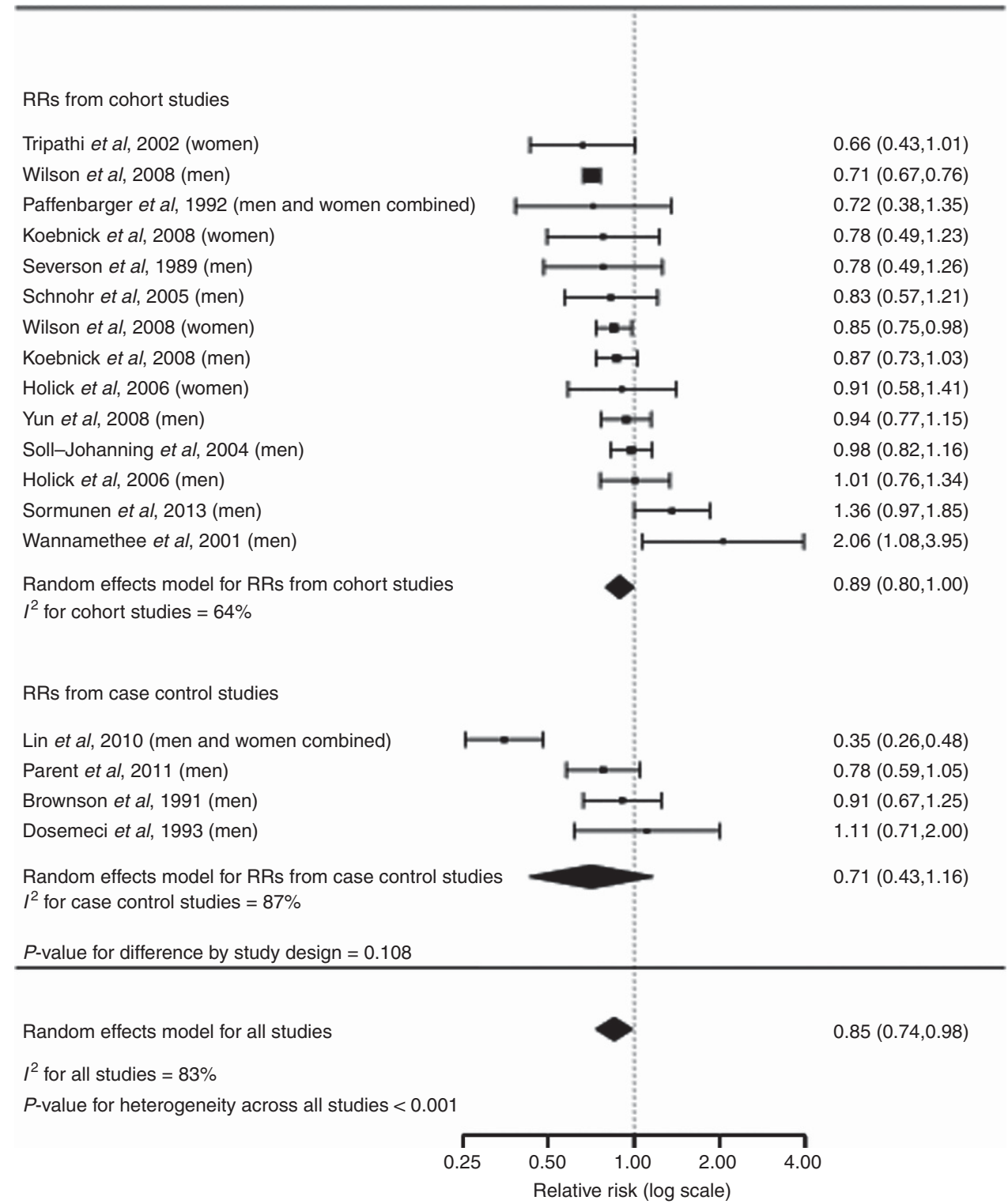

Figure 1. Forest plot quantifying the association between physical activity and bladder cancer risk by study design, including summary risk estimates, $I^{2}$ values, and $P$-value for difference by study design and $P$-value for heterogeneity across all studies. The meta-analysis included 18 bladder cancer risk estimates comparing high vs low levels of physical activity. The $P$-value for difference was estimated from random effects meta-regression comparing a model that included the stratification variable with the null model that did not include the stratification variable.

bladder cancer risk of $10 \%(\mathrm{RR}=0.90,95 \% \mathrm{CI}=0.83-0.97), 14 \%$ $(\mathrm{RR}=0.86,95 \% \quad \mathrm{CI}=0.77-0.96)$, and $17 \% \quad(\mathrm{RR}=0.83,95 \%$ $\mathrm{CI}=0.72-0.95)$, respectively (Figure 3 ).

We conducted a sensitivity analysis in which we omitted one study at a time from the meta-analysis to examine whether the main finding was influenced by a particular study. Removal of individual studies one at a time did not appreciably change the results. In each case, the risk estimate was well within the CIs of the overall risk estimate.

\section{DISCUSSION}

Physical activity and bladder cancer. The findings from this systematic review and meta-analysis show a statistically significant $15 \%$ decreased risk of bladder cancer comparing high $v s$ low levels of physical activity. Our dose-response meta-analysis suggested a linear association between physical activity percentile and bladder cancer risk. We found no strong evidence that the association between physical activity and bladder cancer varied by study design, gender, physical activity intensity, component or measure of physical activity, timing in life of physical activity, type of physical activity assessment, adjustment factors, or study geographic region.

Although smoking is a well-known bladder cancer risk factor (Bachir and Kassouf, 2012) and is associated with physical activity (Kaczynski et al, 2008), adjustment for smoking did not influence the relation of physical activity to bladder cancer in our metaanalysis. This suggests that the mechanisms through which physical activity may protect against bladder cancer are not mediated by the effects of low or no exposure to tobacco smoke among physically active persons. This notion is supported by our finding of no effect modification of the association between physical activity and bladder cancer when we pooled the data from three studies (Koebnick et al, 2008; Yun et al, 2008; Lin et al, 2010) 


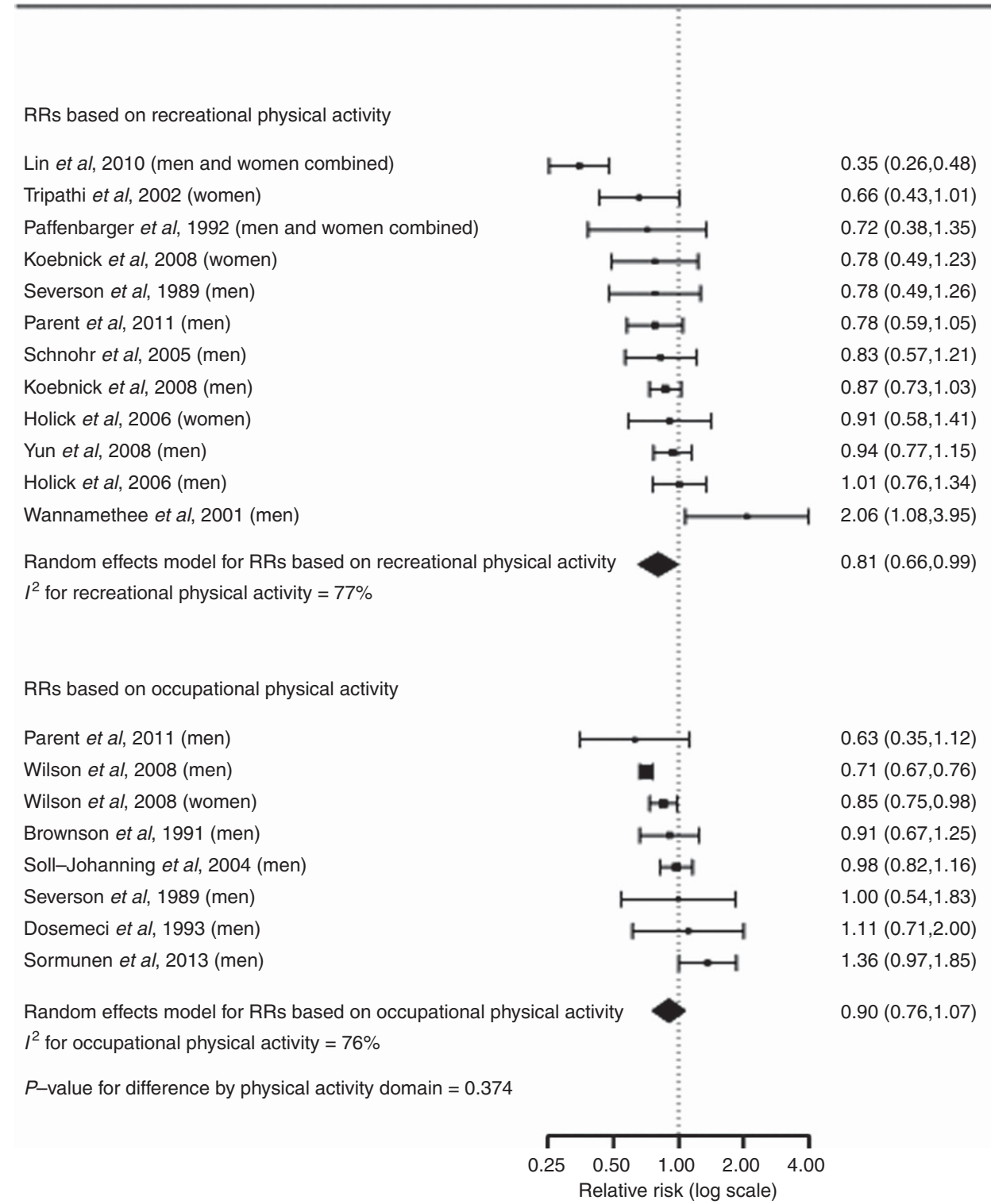

Figure 2. Forest plot quantifying the association between physical activity and bladder cancer risk by domain of physical activity, including summary risk estimates, $I^{2}$ values, and $P$-value for difference by physical activity domain. The meta-analysis included 20 bladder cancer risk estimates comparing high vs low levels of physical activity. The $P$-value for difference was estimated from random effects meta-regression comparing a model that included the stratification variable with the null model that did not include the stratification variable.

that reported on the association between physical activity and bladder cancer stratified by smoking status.

Two previous studies (Holick et al, 2007; Koebnick et al, 2008) investigated whether BMI modified the association between physical activity and bladder cancer. One study (Holick et al, 2007) reported that BMI did not modify the inverse association between physical activity and bladder cancer, whereas the other study (Koebnick et al, 2008) observed a trend of a more pronounced inverse association between physical activity and bladder cancer among lean than among overweight or obese women. This suggests that physical activity and BMI affect bladder cancer risk through distinct biologic pathways. Support for the idea that the apparent protective effect of physical activity on bladder cancer risk is not mediated by healthy body mass among physically active individuals is offered by our finding showing that the magnitude of the risk estimate for physical activity that was adjusted for BMI was similar to the BMI-unadjusted risk estimate.
We did not rely on overall quality scores to stratify risk estimates (Juni et al, 1999). Instead, we assessed relevant methodologic aspects of the underlying studies and explored the influence of those study characteristics on the effect size. Previous meta-analyses of physical activity in relation to cancers of the endometrium (Voskuil et al, 2007), colon (Boyle et al, 2012), pancreas (O'Rorke et al, 2010), and prostate (Liu et al, 2011) found no influence of the methodological quality of the underlying studies on the magnitude of the summary risk estimate.

Although we noted considerable heterogeneity between studies in our main analysis, such heterogeneity was no longer apparent after removal of case-control studies (Brownson et al, 1991; Dosemeci et al, 1993; Lin et al, 2010; Parent et al, 2011) and cohort studies that did not adjust for smoking (Paffenbarger et al, 1992; Soll-Johanning and Bach, 2004; Wilson et al, 2008; Sormunen et al, 2013). Selection and recall bias can be of potential concern in case - control studies and may have contributed to heterogeneity in those studies. It is conceivable that confounding by smoking 


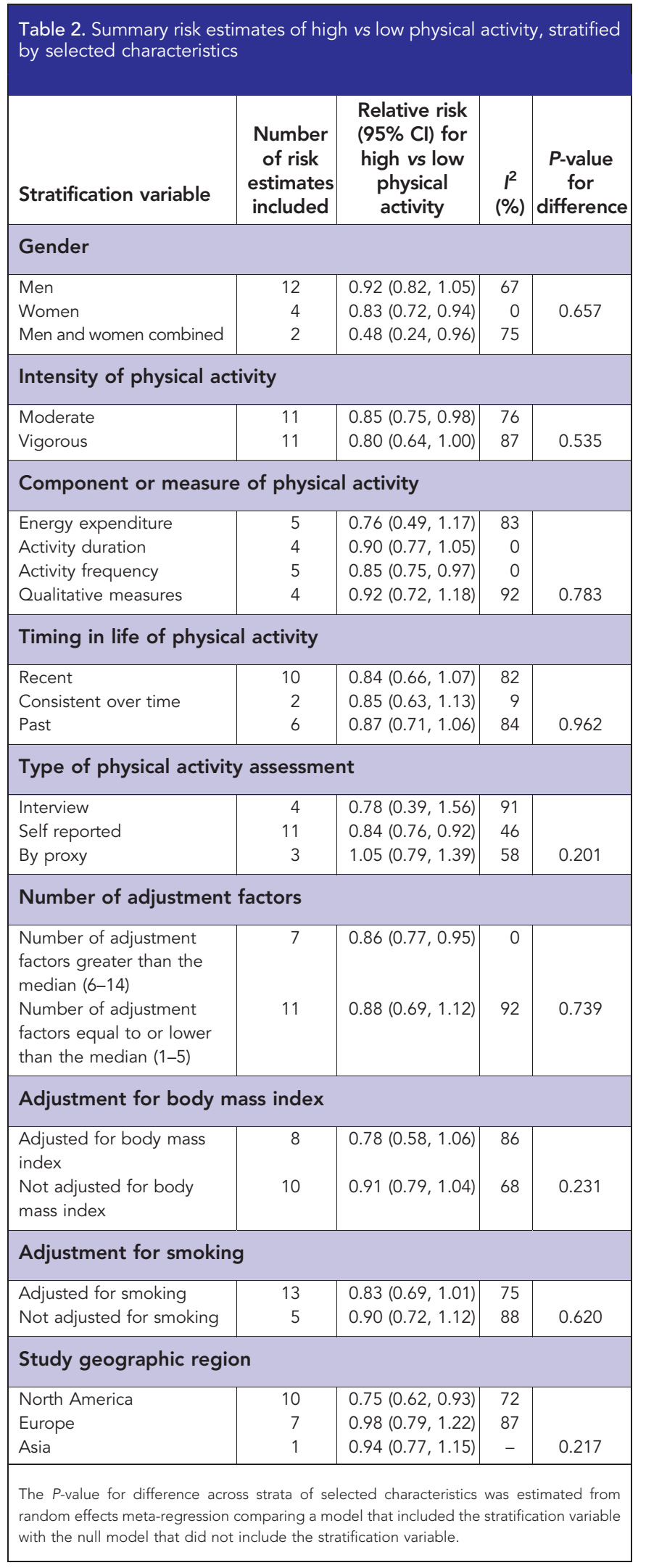

may have been partly responsible for the observed heterogeneity in cohort studies.

Biologic mechanisms. The precise biologic mechanisms through which physical activity may prevent bladder cancer have not been elucidated. However, there is evidence that physical activity increases carcinogenic detoxification, promotes DNA repair

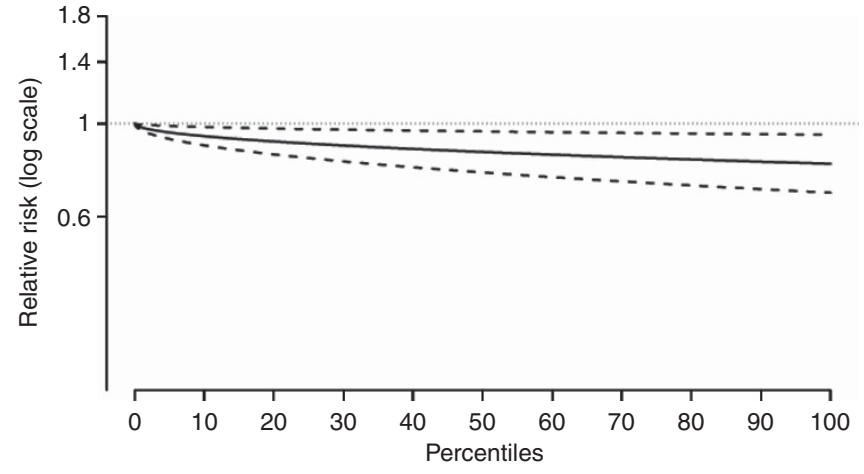

Figure 3. Dose response analysis quantifying the association between increasing percentiles of physical activity and bladder cancer. Analysis includes all studies reporting on three or more physical activity categories.

processes, modifies cell proliferation, differentiation and apoptosis, reduces chronic inflammation, and enhances immune function, factors that are related to carcinogenesis (Rogers et al, 2008). Although not supported by our findings, physical activity may indirectly protect against bladder cancer by reducing smoking (Kaczynski et al, 2008) and preventing adiposity (Wing, 1999; Shaw et al, 2006) and diabetes mellitus (Thomas et al, 2006), three bladder cancer risk factors (Hemelt et al, 2009; Fang et al, 2013; Qin et al, 2013).

Strengths and limitations. One asset of the current study is that it represents the first meta-analysis of the association between physical activity and bladder cancer. Apart from that novelty, one strength of our meta-analysis is the large sample size, which enabled us to conduct a wide range of informative subanalyses, including analyses stratified by physical activity domain and gender. We used standardised criteria to identify relevant articles and abstract pertinent data.

One limitation of our meta-analysis is the variation in the definitions of the reference and exposed groups of physical activity in the underlying studies, ranging from 'no physical activity' to 'less than $5 \mathrm{~h}$ of vigorous physical activity per week' for the reference group to 'physically active' to ' $5 \mathrm{~h}$ of physical activity per week or more' for the exposed group. However, we addressed that issue in a subanalysis by performing a dose-response meta-analysis that was based on comparable percentiles of the distributions of physical activity in the underlying studies. A further shortcoming of our study is that nearly half the studies included in our meta-analysis presented physical activity risk estimates that were adjusted for BMI, which may have represented a certain degree of statistical over-control because the biological pathways linking increased physical activity to decreased bladder cancer risk may in part be mediated by adiposity.

A number of important research gaps persists regarding the relation of physical activity to bladder cancer. Future studies should identify the precise type, duration, frequency, intensity, and timing in life of physical activity relevant for potential decreased risk of bladder cancer. In addition, studies should employ more comprehensive physical activity questionnaires in combination with objective and standardisable instruments, such as accelerometers. In addition, studies among non-Caucasians are needed to elucidate whether results concerning physical activity and bladder cancer are generalisable to those populations.

\section{CONCLUSION}

In conclusion, this meta-analysis suggests that high $v s$ low levels of physical activity are associated with a $15 \%$ decreased risk of 
bladder cancer. Further research is needed to assess the specific duration, frequency, and intensity of physical activity needed for bladder cancer risk reduction. In addition, mechanistic studies are required to clarify the aetiologic pathways through which physical activity may prevent bladder cancer.

\section{ACKNOWLEDGEMENTS}

We thank Dr Helge Knüttel from the library of the University of Regensburg for his assistance in the development of the literature search strategy used in this review.

\section{REFERENCES}

Bachir BG, Kassouf W (2012) Cause-effect? Understanding the risk factors associated with bladder cancer. Expert Rev Anticancer Ther 12: 1499-1502.

Batty GD, Shipley MJ, Kivimaki M, Marmot M, Davey Smith G (2010) Walking pace, leisure time physical activity, and resting heart rate in relation to disease-specific mortality in London: 40 years follow-up of the original Whitehall study. An update of our work with professor Jerry N Morris (1910-2009). Ann Epidemiol 20: 661-669.

Begg CB, Mazumdar M (1994) Operating characteristics of a rank correlation test for publication bias. Biometrics 50: 1088-1101.

Boyle T, Keegel T, Bull F, Heyworth J, Fritschi L (2012) Physical activity and risks of proximal and distal colon cancers: a systematic review and meta-analysis. J Natl Cancer Inst 104: 1548-1561.

Brownson RC, Chang JC, Davis JR, Smith CA (1991) Physical activity on the job and cancer in Missouri. Am J Public Health 81: 639-642.

Dosemeci M, Hayes RB, Vetter R, Hoover RN, Tucker M, Engin K, Unsal M, Blair A (1993) Occupational physical activity, socioeconomic status, and risks of 15 cancer sites in Turkey. Cancer Causes Control 4: 313-321.

Egger M, Davey Smith G, Schneider M, Minder C (1997) Bias in meta-analysis detected by a simple, graphical test. BMJ 315: 629-634.

Fang H, Yao B, Yan Y, Xu H, Liu Y, Tang H, Zhou J, Cao L, Wang W, Zhang J, Zhao L, Chen X, Zhang F, Zhao Y (2013) Diabetes mellitus increases the risk of bladder cancer: an updated meta-analysis of observational studies. Diabetes Technol Ther 15: 914-922.

Ferlay J, Steliarova-Foucher E, Lortet-Tieulent J, Rosso S, Coebergh J, Comber H, Forman D, Bray F (2013) Cancer incidence and mortality patterns in Europe: estimates for 40 countries in 2012. Eur J Cancer 49: 1374-1403.

Hemelt M, Yamamoto H, Cheng KK, Zeegers MP (2009) The effect of smoking on the male excess of bladder cancer: a meta-analysis and geographical analyses. Int J Cancer 124: 412-419.

Higgins JP, Thompson SG (2002) Quantifying heterogeneity in a meta-analysis. Stat Med 21: 1539-1558.

Holick CN, Giovannucci EL, Stampfer MJ, Michaud DS (2007) Prospective study of body mass index, height, physical activity and incidence of bladder cancer in US men and women. Int J Cancer 120: 140-146.

Jemal A, Murray T, Samuels A, Ghafoor A, Ward E, Thun MJ (2003) Cancer statistics, 2003. CA Cancer J Clin 53: 5-26.

Juni P, Witschi A, Bloch R, Egger M (1999) The hazards of scoring the quality of clinical trials for meta-analysis. JAMA 282: 1054-1060.

Kaczynski AT, Manske SR, Mannell RC, Grewal K (2008) Smoking and physical activity: a systematic review. Am J Health Behav 32: 93-110.

Koebnick C, Michaud D, Moore SC, Park Y, Hollenbeck A, Ballard-Barbash R, Schatzkin A, Leitzmann MF (2008) Body mass index, physical activity, and bladder cancer in a large prospective study. Cancer Epidemiol Biomarkers Prev 17: 1214-1221.

Lin J, Wang J, Greisinger AJ, Grossman HB, Forman MR, Dinney CP, Hawk ET, Wu X (2010) Energy balance, the PI3K-AKT-mTOR pathway genes, and the risk of bladder cancer. Cancer Prev Res 3: 505-517.

Liu Y, Hu F, Li D, Wang F, Zhu L, Chen W, Ge J, An R, Zhao Y (2011) Does physical activity reduce the risk of prostate cancer? A systematic review and meta-analysis. Eur Urol 60: 1029-1044.
Moher D, Liberati A, Tetzlaff J, Altman DG (2009) Preferred reporting items for systematic reviews and meta-analyses: the PRISMA statement. BMJ 339: b2535.

O'Rorke MA, Cantwell MM, Cardwell CR, Mulholland HG, Murray LJ (2010) Can physical activity modulate pancreatic cancer risk? a systematic review and meta-analysis. Int J Cancer 126: 2957-2968.

Paffenbarger Jr RS, Lee IM, Wing AL (1992) The influence of physical activity on the incidence of site-specific cancers in college alumni. Adv Exp Med Biol 322: 7-15.

Parent ME, Rousseau MC, El-Zein M, Latreille B, Desy M, Siemiatycki J (2011) Occupational and recreational physical activity during adult life and the risk of cancer among men. Cancer Epidemiol 35: 151-159.

Pukkala E, Kaprio J, Koskenvuo M, Kujala U, Sarna S (2000) Cancer incidence among Finnish world class male athletes. Int J Sports Med 21: 216-220.

Qin Q, Xu X, Wang X, Zheng XY (2013) Obesity and risk of bladder cancer: a meta-analysis of cohort studies. Asian Pac J Cancer Prev 14: 3117-3121.

R Development Core Team (2011) R: A Language and Environment for Statistical Computing, Vienna, Austria.

Rogers CJ, Colbert LH, Greiner JW, Perkins SN, Hursting SD (2008) Physical activity and cancer prevention: pathways and targets for intervention. Sports Med 38: 271-296.

Rota M, Bellocco R, Scotti L, Tramacere I, Jenab M, Corrao G, La Vecchia C, Boffetta P, Bagnardi V (2010) Random-effects meta-regression models for studying nonlinear dose-response relationship, with an application to alcohol and esophageal squamous cell carcinoma. Stat Med 29: 2679-2687.

Schnohr P, Gronbaek M, Petersen L, Hein HO, Sorensen TI (2005) Physical activity in leisure-time and risk of cancer: 14-year follow-up of 28,000 Danish men and women. Scand J Public Health 33: 244-249.

Severson RK, Nomura AM, Grove JS, Stemmermann GN (1989) A prospective analysis of physical activity and cancer. Am J Epidemiol 130: 522-529.

Shaw K, Gennat H, O’Rourke P, Del Mar C (2006) Exercise for overweight or obesity. Cochrane Database Syst Rev 4: CD003817.

Siegel R, Naishadham D, Jemal A (2013) Cancer statistics, 2013. CA Cancer J Clin 63: 11-30.

Soll-Johanning H, Bach E (2004) Occupational exposure to air pollution and cancer risk among Danish urban mail carriers. Int Arch Occup Environ Health 77: 351-356.

Sormunen J, Backmand HM, Sarna S, Kujala UM, Kaprio J, Dyba T, Pukkala E (2013) Lifetime physical activity and cancer incidence-A cohort study of male former elite athletes in Finland. J Sci Med Sport S1440-2440: 00470-00472.

Thomas DE, Elliott EJ, Naughton GA (2006) Exercise for type 2 diabetes mellitus. Cochrane Database Syst Rev 3: CD002968.

Tripathi A, Folsom AR, Anderson KE (2002) Risk factors for urinary bladder carcinoma in postmenopausal women. The Iowa Women's Health Study. Cancer 95: 2316-2323.

Viechtbauer W (2010) Conducting Meta-Analyses in R with the metafor Package. J Stat Software 36: 1-48.

Voskuil DW, Monninkhof EM, Elias SG, Vlems FA, Van Leeuwen FE (2007) Physical activity and endometrial cancer risk, a systematic review of current evidence. Cancer Epidemiol Biomarkers Prev 16: 639-648.

Wannamethee SG, Shaper AG, Walker M (2001) Physical activity and risk of cancer in middle-aged men. Br J Cancer 85: 1311-1316.

Wilson RT, Donahue M, Gridley G, Adami J, El Ghormli L, Dosemeci M (2008) Shared occupational risks for transitional cell cancer of the bladder and renal pelvis among men and women in Sweden. Am J Ind Med 51: 83-99.

Wing RR (1999) Physical activity in the treatment of the adulthood overweight and obesity: current evidence and research issues. Med Sci Sports Exerc 31: S547-S552.

Yun YH, Lim MK, Won YJ, Park SM, Chang YJ, Oh SW, Shin SA (2008) Dietary preference, physical activity, and cancer risk in men: national health insurance corporation study. BMC Cancer 8: 366.

This work is published under the standard license to publish agreement. After 12 months the work will become freely available and the license terms will switch to a Creative Commons AttributionNonCommercial-Share Alike 3.0 Unported License.

Supplementary Information accompanies this paper on British Journal of Cancer website (http://www.nature.com/bjc) 